\title{
Rheumatherapie in Corona-Zeiten
}

Während die ersten Empfehlungen der Deutschen Gesellschaft für Rheumatologie e.V. (DGRh) sich noch auf allgemeine Erkenntnisse zur Infektanfälligkeit von Rheumapatienten stützen mussten, liegen inzwischen Fallberichte, Registerdaten und erste Studien vor, die detailliertere Aussagen zu den Erkrankungsrisiken dieser Patientengruppe erlauben. Die neuen Erkenntnisse haben nun Eingang in eine aktualisierte Fassung der Therapieempfehlungen gefunden.

Auf den ersten Blick gibt die neue Empfehlung weitgehend Entwarnung: Rheumapatienten haben den bisher zusammengetragenen Daten zufolge kein erhöhtes Risiko, sich mit SARS-CoV-2 anzustecken. „Auch das Risiko für einen schweren Verlauf von COVID-19 scheint nicht erhöht zu sein", sagt Prof. Dr. Hendrik Schulze-Koops, Präsident der DGRh und Leiter der Rheumaeinheit am Klinikum der LMU München. Doch gelte diese Entwarnung mit Einschränkungen: Einige der bekannten, allgemeinen Risikofaktoren für schwere Verläufe wie ein höheres Lebensalter, männliches Geschlecht, Rauchen, Diabetes, starkes Übergewicht oder vorbestehende Lungen-, Herz- oder Nierenerkrankungen seien bei Rheumapatienten häufiger als im Bevölkerungsdurchschnitt. Sie dürften daher nicht außer Acht gelassen werden. Durch die entzündlich-rheumatische Erkrankung selbst scheint das Corona-Risiko dagegen nur dann erhöht zu sein, wenn die Patienten Glukokortikoide („Cortison“) in einer Dosis von 10 Milligramm und mehr pro Tag einnehmen oder wenn die Erkrankung gerade besonders aktiv ist, die Rheumasymptome also schlecht medikamentös beherrscht werden können.

In ihren zehn Kernempfehlungen wiederholen die Experten daher ihren Rat, eine gut eingestellte Rheumamedikation nicht allein aus Sorge vor COVID-19 zu verändern. „Ein Umstellen der Medikation geht oft mit einem Aufflammen der rheumatologischen Grunderkrankung einher und erhöht das COVID-19 Risiko dann erst recht", erklärt Prof. Dr. Christof Specker, Direktor der Klinik für Rheumatologie und Klinische Immunologie am Klinikum Essen-Mitte und Sprecher der Ad-hoc Kommission COVID-19-Register der DGRh. Lediglich bei Patienten mit positivem SARS-CoV-2-Abstrich und/oder bereits beginnenden COVID19-Symptomen solle die Therapie mit Biologika und bestimmten anderen immunmodulierenden Rheumamedikamenten unterbrochen werden. Eine Therapie mit Glukokortikoiden unter 10 Milligramm pro Tag könne dagegen fortgesetzt werden.

Besonderes Augenmerk richten die Experten darauf, Alltag und Versorgung der Patienten nicht unnötig einzuschränken. In der Regel sei es nicht gerechtfertigt, Rheumapatienten allein aufgrund einer angenommenen Gefährdung durch COVID-19 Arbeitsunfähigkeit zu attestieren, heißt es in den Empfehlungen. Auch dürfe keinesfalls auf medizinisch notwendige Eingriffe und Therapien verzichtet werden. Selbstverständlich sollten auch Rheuma-Patienten sich an die allgemein geltenden Hygieneund Abstandsregeln halten, außerdem empfiehlt die DGRh die Benutzung der Corona-Warn-App.

Auch wenn die Datenbasis im Vergleich zum Frühjahr bedeutend breiter geworden ist - allein im DGRh-eigenen COVID-19-Register sind inzwischen mehr als 350 Krankheitsverläufe erfasst - machen die Experten darauf aufmerksam, dass die Evidenz noch keineswegs so belastbar ist wie sonst bei Behandlungsempfehlungen üblich.„Es liegt bei einem so jungen und dynamischen Geschehen wie der COVID-
19-Pandemie in der Natur der Sache, dass zum Beispiel noch keine randomisiert-kontrollierten Studien vorliegen“, sagt Specker. Um dem beständigen Eintreffen neuer Erkenntnisse Rechnung zu tragen, wird die DGRh ihre Empfehlungen auch weiterhin regelmäßig aktualisieren und auf ihrer Homepage und in Fachmedien veröffentlichen.

\section{Literatur}

1. Schulze-Koops $\mathrm{H}$, Iking-Konert $\mathrm{C}$, Leipe J et al (2020) Handlungsempfehlungen der Deutschen Gesellschaft für Rheumatologie e.V. für die Betreuung von Patienten mit entzündlich rheumatischen Erkrankungen im Rahmen der SARS-CoV-2/COVID-19-Pandemie - Update Juli 2020. Z Rheumatol. https://doi.org/10.1007/s00393-020-00851-x

Hinweis des Verlags. Der Verlag bleibt in Hinblick auf geografische Zuordnungen und Gebietsbezeichnungen in veröffentlichten Karten und Institutsadressen neutral.

rheuma plus $2020 \cdot 19: 164-165$ https://doi.org/10.1007/s12688020-00374-y

(c) Springer-Verlag GmbH Austria, ein Teil von Springer Nature 2020 
Hier steht eine Anzeige.

Springer 